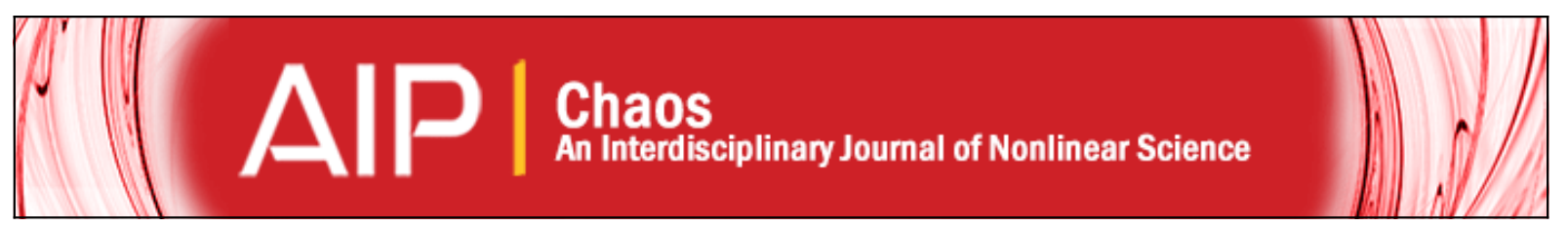

\title{
Modulational instability of co-propagating internal wavetrains under rotation
}

A. J. Whitfield and E. R. Johnson

Citation: Chaos: An Interdisciplinary Journal of Nonlinear Science 25, 023109 (2015); doi: 10.1063/1.4908571

View online: http://dx.doi.org/10.1063/1.4908571

View Table of Contents: http://scitation.aip.org/content/aip/journal/chaos/25/2?ver=pdfcov

Published by the AIP Publishing

\section{Articles you may be interested in}

Rotation-induced nonlinear wavepackets in internal waves

Phys. Fluids 26, 056606 (2014); 10.1063/1.4879075

On a plasma having nonextensive electrons and positrons: Rogue and solitary wave propagation

Phys. Plasmas 18, 082306 (2011); 10.1063/1.3620411

Effect of second ion temperature on modulation stability of dust-acoustic solitary waves with dust charge variation

Phys. Plasmas 14, 103704 (2007); 10.1063/1.2784765

Saddle-node bifurcation and modulational instability associated with the pulse propagation of dust ion-acoustic waves in a viscous dusty plasma: A complex nonlinear Schrödinger equation

Phys. Plasmas 14, 012110 (2007); 10.1063/1.2409493

Modulational instability of two colliding whistlers

Phys. Plasmas 11, 1233 (2004); 10.1063/1.1643921

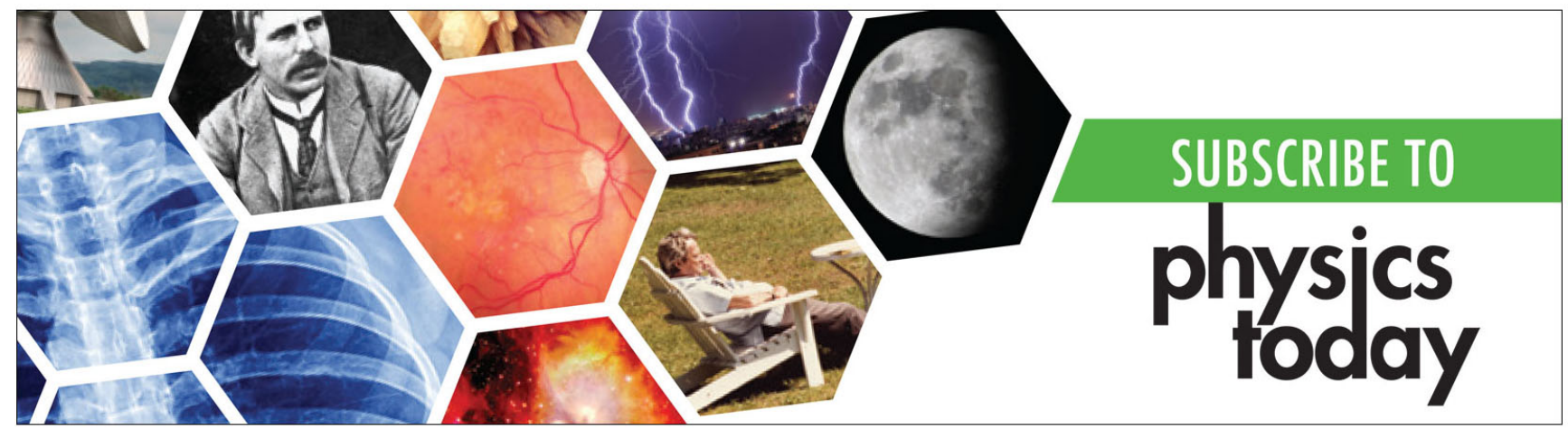




\title{
Modulational instability of co-propagating internal wavetrains under rotation
}

\author{
A. J. Whitfield ${ }^{\text {a) }}$ and E. R. Johnson ${ }^{\text {b) }}$ \\ Department of Mathematics, University College London, London WC1E 6BT, United Kingdom
}

(Received 23 July 2014; accepted 4 February 2015; published online 13 February 2015)

\begin{abstract}
Weakly-nonlinear unidirectional long internal waves in a non-rotating frame are well described by the Korteweg-de Vries equation (KdV). Within the KdV framework, all isolated monochromatic wavetrains are stable to modulational instability. However, analysis of a coupled nonlinear Schrödinger equation system (CNLS) has shown that all systems of two co-propagating monochromatic wavetrains in the $\mathrm{KdV}$ are modulationally unstable. To take into account the effect of the background rotation of the Earth on long internal waves, this analysis is extended here to derive the CNLS for the rotation-modified KdV, or Ostrovsky, equation. Rotation stabilises wavetrain pairs when the wavelengths of both waves comprising the wavetrains are longer than the linear wave with maximum group velocity. The particular case when the wavetrains have different wavenumbers but the same linear group speed is emphasised. (C) 2015 AIP Publishing LLC.

[http://dx.doi.org/10.1063/1.4908571]
\end{abstract}

A periodic train of waves of fixed, short wavelength on the surface of the ocean is unstable to large-scale modulational, or Benjamin-Feir, instability. In contrast, long waves, both on the surface and internal, in shallow seas can be accurately modelled by the Korteweg-de Vries equation ( $K d V)$ and a single wavetrain in the $K d V$ is modulationally stable. It has been shown, however, that the presence of a second co-propagating wavetrain in the shallow-water, KdV limit renders the otherwise stable flow unstable with consequences for the growth of freak waves on swell. One effect that has not been considered in this context is the background rotation of the Earth. Internal waves can persist over many inertial periods allowing background rotation to become significant. This paper shows that background rotation can stabilise copropagating wavetrains that are unstable when rotation is neglected. The bases of the analysis are coupled nonlinear Schrödinger equations like those for the $\mathrm{KdV}$, but derived for co-propagating wavetrains in the rotationmodified KdV, or Ostrovsky, equation. The analysis also has implications for the initial value problem for the Ostrovsky equation.

\section{INTRODUCTION}

Oceanic internal waves are often assumed to have amplitudes small compared with the ocean depth (weak nonlinearity) and wavelengths long compared with the depth (weak dispersion). Korteweg-de Vries (KdV) theory then gives an accurate description of the shape and speed of observed waves. ${ }^{1-4}$ In the highly nonlinear large-amplitude regime, $\mathrm{KdV}$-type theories have even been used with some success to model internal waves outside their formal range of validity ${ }^{5}$ with the addition of a higher-order cubic nonlinear term. Over the past two decades, strongly nonlinear

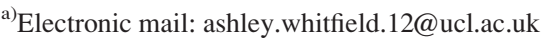

${ }^{\text {b) Electronic mail: e.johnson@ucl.ac.uk }}$
}

models have also been considered. ${ }^{3}$ Rotation is often taken to be negligible in these theories although observed waves can persist for several days allowing rotational effects to become important. ${ }^{6,7}$ The simplest weakly-nonlinear model taking the effects of rotation into account within this framework is the Ostrovsky (rotation-modified KdV) equation, ${ }^{8}$ and one consequence of rotational effects is the terminal decay of the otherwise persistent KdV solitary wave. ${ }^{9}$ When amplitudes are small and rotation is dominant, the soliton rapidly breaks into two co-propagating near-linear inertiagravity wavetrains with distinct local wavenumbers ${ }^{10}$ and it is interactions between such wavetrains that are considered here. Interactions between modes with different vertical structure, leading to coupled Ostrovsky equations, have been considered recently in the context of an initial value problem for coupled Ostrovsky equations with ${ }^{11}$ and without ${ }^{12}$ a background shear flow, but the problem of two long trains of waves with the same vertical structure but different horizontal wavenumbers, leading to coupled nonlinear Schrödinger equations (CNLS), appears not to have been discussed.

Historically, the propagation of multiple wavetrains in oceanography has received little attention compared with the single wavetrain problem, but a report by Thompson ${ }^{13}$ revealed that approximately $65 \%$ of wavetrains near coasts of the United states contain two or more separate peaks in the frequency domain. Onorato et al. ${ }^{14}$ found from a nonlinear Schrödinger equation (NLS) analysis of the KdV that the presence of a second co-propagating wavetrain drastically alters the stability characteristics of the wave modulation, with the inter-wavetrain interaction through the mean flow term making the usually modulationally stable $\mathrm{KdV}$ equation unstable. This instability is commonly described as modulational instability (MI), sideband instability, or even Benjamin-Feir instability, after the pioneering work of Benjamin and Feir ${ }^{15}$ on monochromatic surface water waves. There appears to be no equivalent analysis for the Ostrovsky equation. Since co-propagating inertia-gravity wavetrains appear naturally in the initial value problem for 
the Ostrovsky equation, ${ }^{10}$ the question arises as to whether the presence of a second wavetrain affects the MI characteristics of the Ostrovsky equation. This paper thus investigates the interaction of co-propagating wavetrains, with the same vertical structure, under rotation.

Section II A derives briefly a general coupled NLS system for two co-propagating wavetrains of different linear group speed and shows that the zero-mass constraint on solutions of the Ostrovsky equation eliminates the mean flow term in the coupled equations. Section II B gives the corresponding dispersion relation for modulated waves. Section IIC verifies by direct comparison with integrations of the full Ostrovsky equations, the predictions of Secs. II A and II B. Finally, Sec. III considers the case where both wavetrains have distinct wavenumbers but the same linear group speed, a situation impossible for the $\mathrm{KdV}$ in the absence of rotation.

\section{COUPLED NONLINEAR SCHRÖDINGER EQUATION}

\section{A. A brief derivation}

The one-dimensional horizontal structure of a single vertical internal mode is governed by the Ostrovsky equation $^{8}$ which can be written, relative to a frame moving at linear long wave speed, as

$$
\left(\eta_{t}+\alpha \eta \eta_{x}+\beta \eta_{x x x}\right)_{x}=\gamma \eta,
$$

where $\eta$ is the interfacial displacement, $\alpha$ the strength of weak nonlinearity, $\beta$ weak non-hydrostatic dispersion and $\gamma$ the strength of weak rotation, reducing to the $\mathrm{KdV}$ for $\gamma=0$. For the case of oceanic internal waves, it can also be assumed without loss of generality that $\alpha, \beta, \gamma>0 .{ }^{16}$ The interest here, as in Onorato et al., ${ }^{14}$ is in the stability of two wavetrains, propagating in the same direction, centred at wavenumbers $k_{1}$ and $k_{2}$, where "centred" refers to narrowbanded spectra, i.e., $\delta k_{i} / k_{i} \ll 1\left(i=1,2\right.$, for $\delta k_{i}$ the characteristic width of the spectra). CNLS for the Ostrovsky equation describing the evolution of two co-propagating wavetrains can be derived following the approach in Onorato et al. ${ }^{14}$ for the $\mathrm{KdV}$, and so details are kept brief here. Introduce the slow space $X=\epsilon x$ and time $T=\epsilon t$ variables, where $\epsilon \ll 1$, the fast phase variable $\theta=k x-\omega t$, and the expansion around wavenumbers $k_{1}$ and $k_{2}$

$$
\begin{aligned}
\eta= & \epsilon\left(A \exp \left[\mathrm{i} \theta_{1}\right]+\mathrm{B} \exp \left[\mathrm{i} \theta_{2}\right]\right)+\epsilon^{2}\left(A_{2} \exp \left[2 \mathrm{i} \theta_{1}\right]\right. \\
& +\mathrm{B}_{2} \exp \left[2 \mathrm{i} \theta_{2}\right]+C_{2} \exp \left[\mathrm{i}\left(\theta_{1}+\theta_{2}\right)\right] \\
& \left.+\mathrm{D}_{2} \exp \left[\mathrm{i}\left(\theta_{1}-\theta_{2}\right)\right]+\mathrm{M}\right)+\epsilon^{3}\left(A_{3} \exp \left[3 \mathrm{i} \theta_{1}\right]\right. \\
& +\mathrm{B}_{3} \exp \left[3 \mathrm{i} \theta_{2}\right]+C_{3} \exp \left[\mathrm{i}\left(2 \theta_{1}+\theta_{2}\right)\right] \\
& +\mathrm{D}_{3} \exp \left[\mathrm{i}\left(2 \theta_{1}-\theta_{2}\right)\right]+F_{3} \exp \left[\mathrm{i}\left(\theta_{1}+2 \theta_{2}\right)\right] \\
& \left.+\mathrm{G}_{3} \exp \left[\mathrm{i}\left(\theta_{1}-2 \theta_{2}\right)\right]\right)+ \text { c.c...., }
\end{aligned}
$$

where c.c. denotes the complex conjugate of the preceding terms, and $A, A_{2}, A_{3}, B, B_{2}, B_{3}, C_{2}, C_{3}, D_{2}, D_{3}, F_{3}, G_{3}$, and $M$ are all functions of the slow variables. Substituting (2) into (1) - examining each order in $\epsilon$, imposing periodicity of the solution, and therefore setting the coefficients of the harmonics at each order to zero-leads to a closed set of equations for the leading order terms $A$ and $B$. Equating secular terms at order $\epsilon$ gives the linear dispersion relation of the Ostrovsky equation, $\omega_{i}=\gamma / k_{i}-\beta k_{i}^{3}$. At order $\epsilon^{2}$, the second harmonics give

$$
\begin{aligned}
& A_{2}=2 \alpha k_{1}^{2} A^{2} /\left(12 \beta k_{1}^{4}+3 \gamma\right), \\
& B_{2}=2 \alpha k_{2}^{2} B^{2} /\left(12 \beta k_{2}^{4}+3 \gamma\right), \\
& C_{2}=\frac{\alpha\left(k_{1}+k_{2}\right)^{2} A B}{\gamma\left(k_{2} / k_{1}+k_{1} / k_{2}+1\right)+3 \beta k_{1} k_{2}\left(k_{1}+k_{2}\right)^{2}}, \\
& D_{2}=\frac{-\alpha\left(k_{1}-k_{2}\right)^{2} A B^{*}}{\gamma\left(k_{2} / k_{1}+k_{1} / k_{2}-1\right)+3 \beta k_{1} k_{2}\left(k_{1}-k_{2}\right)^{2}} .
\end{aligned}
$$

As in the derivation of the CNLS for the $\mathrm{KdV},{ }^{14}$ some higherorder terms in the expansion introduce factors proportional to $\left(k_{1}-k_{2}\right)^{-2}$ and so the expansion becomes disordered for $\left|k_{1}-k_{2}\right|$ sufficiently small. It is shown through direct numerical integrations in Sec. IIC below that this restriction is not significant in practice. Using relations (3), neglecting terms higher-order than $\epsilon^{3}$ and equating terms from the first harmonics gives the evolution equations for $A$ and $B$

$$
\begin{aligned}
& \mathrm{i}\left(A_{T}+c_{1} A_{X}\right)+\epsilon\left[\Delta_{1} A_{X X}+\left(\mu_{1}|A|^{2}+\sigma_{1}|B|^{2}\right) A\right]=0, \\
& \mathrm{i}\left(B_{T}+c_{2} B_{X}\right)+\epsilon\left[\Delta_{2} B_{X X}+\left(\mu_{2}|B|^{2}+\sigma_{2}|A|^{2}\right) B\right]=0,
\end{aligned}
$$

where

$$
\begin{aligned}
c_{i}= & \partial \omega_{i} / \partial k_{i}=-3 \beta k_{i}^{2}-\gamma / k_{i}^{2}, \\
\Delta_{i}= & \frac{1}{2} \partial c_{i} / \partial k_{i}=\frac{1}{2} \partial^{2} \omega_{i} / \partial k_{i}^{2}=-3 \beta k_{i}+\gamma / k_{i}^{3}, \\
\mu_{i}= & -2 \alpha^{2} k_{i}^{3} /\left(12 \beta k_{i}^{4}+3 \gamma\right), \\
\sigma_{i}= & -2 \alpha^{2} \gamma k_{i}\left(k_{1}+k_{2}\right)^{2} /\left\{\gamma^{2}\left[\left(k_{2} / k_{1}+k_{1} / k_{2}\right)^{2}-1\right]\right. \\
& \left.+9 \beta^{2} k_{1}^{2} k_{2}^{2}\left(k_{1}^{2}-k_{2}^{2}\right)^{2}+6 \beta \gamma\left(k_{1}^{4}+k_{2}^{4}\right)\right\} .
\end{aligned}
$$

Here, $c_{i}$ is the linear group velocity of wave $k_{i}$ with derivative $2 \Delta_{i}, \mu_{i}$ is the usual nonlinear frequency correction, ${ }^{16}$ and $\sigma_{i}$ is the interaction term. The group velocity $c_{i}$ has a maximum when $k_{i}=(\gamma / 3 \beta)^{1 / 4}=k_{c}$ (say). Then $\Delta_{i}<0$ for $k_{i}>k_{c}$ and $\Delta_{i}>0$ for $k_{i}<k_{c}$. For a wavetrain $i$ in isolation, it is well known that if $\Delta_{i}$ has the same sign as $\mu_{i}$, the wavetrain is modulationally unstable. ${ }^{17}$ Since $\mu_{i}<0$, an isolated wavetrain is modulationally unstable for $k_{i}>k_{c}$ and stable for $k_{i}<k_{c}{ }^{16}{ }^{16}$ This paper extends these results to the coupled wavetrain.

Now, $\sigma_{1,2}$ vanish for $\gamma=0$, and so the modes appear to decouple in the KdV limit. This is because Eq. (1) requires that for any $\gamma \neq 0$, the total mass $\int_{-\infty}^{\infty} \eta \mathrm{d} x$ of any solution of the Ostrovsky equation vanishes, removing the mean flow term $M$ that gives the coupling in the CNLS for the KdV. The derivation of (4) implicitly requires that $\gamma$ is of order unity or larger and the CNLS for $\gamma$ of higher order in $\epsilon$ is of different (non-local) form which does indeed reduce to the CNLS for the KdV when $\gamma=0$. The mass constraint also weakens greatly the coupling in the Ostrovsky equation. Fig. 1 shows $\sigma_{1}$ as a function of $k_{2}$ for $k_{1}=2$ when $\gamma=\beta=1$. The interaction is strongest when the wavenumbers are comparable and weakens as $\left|k_{1}-k_{2}\right|$ increases. 


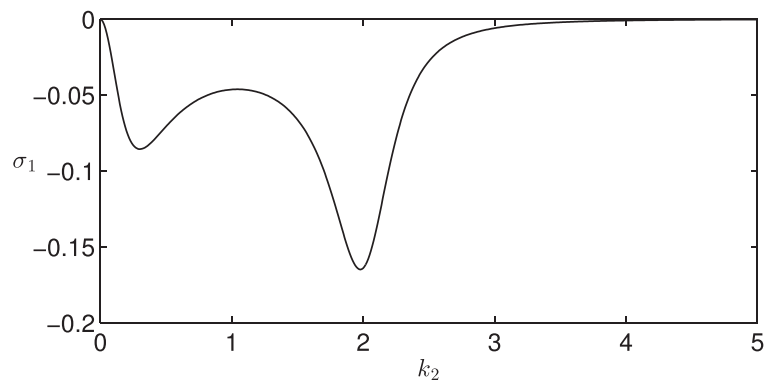

FIG. 1. The interaction coefficient $\sigma_{1}$ in (4) as a function of $k_{2}$ for $k_{1}=2$ when $\gamma=\beta=1$. The interaction is strongest when the wavetrain carrier wavenumbers are comparable and weakens as $\left|k_{1}-k_{2}\right|$ increases.

Equation (4) should be independent of $\epsilon$ to be consistent, although - as noted in Onorato et al. ${ }^{14}$ - this is not always possible for the CNLS. The factor $\epsilon$ is removed in the derivation of the NLS for a single wavetrain by moving to a reference frame translating at the linear group velocity. This is not possible in general for the CNLS as it requires wavenumbers $k_{1}$ and $k_{2}$ to be chosen such that $c_{1}=c_{2}$, although Sec. III below gives an important example where $c_{1}$ does indeed equal $c_{2}$. First, to retain generality, the approach of Onorato et $a l .{ }^{14}$ is followed by introducing a further scaling of the space $\chi=\epsilon X$ and time $\tau=\epsilon T$ variables. Equation (4) can then be re-written as

$$
\begin{aligned}
& \mathrm{i}\left(A_{\tau}+c_{1} A_{\chi}\right)+\epsilon^{2} \Delta_{1} A_{\chi \chi}+\left(\mu_{1}|A|^{2}+\sigma_{1}|B|^{2}\right) A=0, \\
& \mathrm{i}\left(B_{\tau}+c_{2} B_{\chi}\right)+\epsilon^{2} \Delta_{2} B_{\chi \chi}+\left(\mu_{2}|B|^{2}+\sigma_{2}|A|^{2}\right) B=0 .
\end{aligned}
$$

Onorato et al. ${ }^{14}$ take (5) as the basis for discussion of the $\mathrm{KdV}$ problem, and so it is taken here as the basis for discussing MI for a general wavetrain pair in the Ostrovsky equation. In an examination of two-wave and four-wave MI in the context of the nonlinear Klein-Gordon equation, Griffiths, Grimshaw, and Khusnutdinova ${ }^{18}$ discuss this approach and note that although equations of the form (5) are not generally asymptotically exact, in that they contain small dispersive terms which are strictly beyond the leading order transport terms, they can be useful for diagnosing MI as they do capture the leading order dispersive and nonlinear effects. The validity of this approach for the Ostrovsky equation is confirmed in Sec. IIC below by direct comparison of growth rates obtained from (5) with those from numerical integration of the full Ostrovsky equation.

\section{B. Dispersion relation for modulational waves}

Following Onorato et al., ${ }^{14}$ consider the finite amplitude plane wave solutions of (5) given by

$$
\begin{gathered}
A=\tilde{A} \exp \left(-\mathrm{i} \omega_{A} \tau\right), \quad B=\tilde{B} \exp \left(-\mathrm{i} \omega_{B} \tau\right) \\
\omega_{A}=-\left(\mu_{1}|\tilde{A}|^{2}+\sigma_{1}|\tilde{B}|^{2}\right), \quad \omega_{B}=-\left(\mu_{2}|\tilde{B}|^{2}+\sigma_{2}|\tilde{A}|^{2}\right),
\end{gathered}
$$

and take these to be perturbed to

$$
\begin{aligned}
& A=\tilde{A}(1+a) \exp \left(-\mathrm{i} \omega_{A} \tau+\phi_{A}\right), \\
& B=\tilde{B}(1+b) \exp \left(-\mathrm{i} \omega_{B} \tau+\phi_{B}\right),
\end{aligned}
$$

where $a, b$ and $\phi_{A}, \phi_{B}$ are small perturbations in amplitude and phase, respectively. Linearizing the perturbation equations and looking for wavelike solutions where $a, b$, $\phi_{A}, \phi_{B} \propto \exp [\mathrm{i}(K \chi-\Omega \tau)]$ gives the dispersion relation $\Omega=\Omega(K)$ for the modulation frequency $\Omega$ in terms of the modulation wavenumber $K$, as the monic quartic polynomial

$$
\tilde{D}_{1}(\Omega, K) \tilde{D}_{2}(\Omega, K)=4 \epsilon^{4} \sigma_{1} \sigma_{2} \Delta_{1} \Delta_{2}|\tilde{A}|^{2}|\tilde{B}|^{2} K^{4},
$$

where

$$
\begin{aligned}
& \tilde{D}_{1}(\Omega, K)=\left(\Omega-K c_{1}\right)^{2}+\epsilon^{2} \Delta_{1} K^{2}\left(2 \mu_{1}|\tilde{A}|^{2}-\epsilon^{2} \Delta_{1} K^{2}\right), \\
& \tilde{D}_{2}(\Omega, K)=\left(\Omega-K c_{2}\right)^{2}+\epsilon^{2} \Delta_{2} K^{2}\left(2 \mu_{2}|\tilde{B}|^{2}-\epsilon^{2} \Delta_{2} K^{2}\right),
\end{aligned}
$$

give the monic quadratic dispersion relations $\tilde{D}_{i}(\Omega, K)=0$ for each wavetrain in isolation. Since the coefficients are real, any complex roots for $\Omega$ occur in conjugate pairs and so the coupled wavetrains are modulationally unstable for any parameters values, where (6a) has at least one complex root for any $K$ and stable only for parameters such that all four roots of (6a) are real for all $K$. Fig. 2 shows the maximum growth rate (the maximum over the four roots of the imaginary part of $\Omega)$ in $\left(K, k_{2}\right)$ space for two values of $k_{1}$ with $\epsilon=0.1$ and all other parameters unity. The white regions show where the maximum growth rate is zero and so correspond to stable waves while the shaded regions give unstable waves. When both $k_{1,2}<k_{c}$, the system is stable as given by the white region for all $K$ of Fig. 2(a), and when $k_{1}>k_{c}$, in Fig. 2(b), the system is always unstable as the growth rate is nonzero for some $K$.
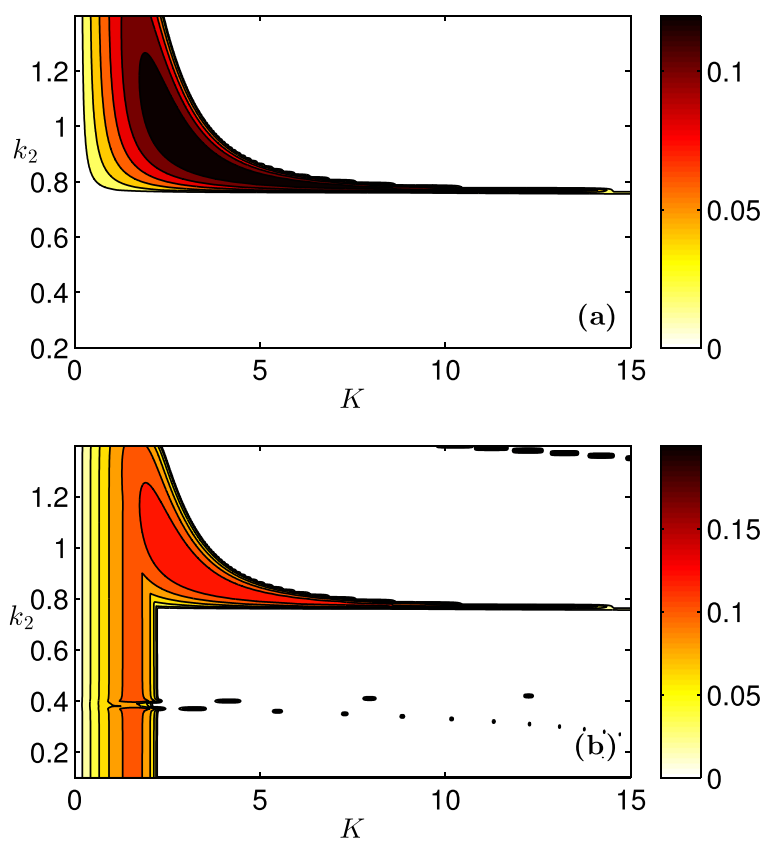

FIG. 2. The maximum growth rate $[\Im m(\Omega)]_{\max }$ contoured in $\left(K, k_{2}\right)$ space for a modulation of wavenumber $K$ to two wavetrains of wavenumbers $k_{1}$ and $k_{2}$ calculated from the dispersion relation (6) for parameters $\epsilon=0.1$ and $\tilde{A}, \tilde{B}$, $\alpha, \beta, \gamma=1$. (a) $k_{1}=0.1<k_{c}$ (stable). The entire region $k_{2}<k_{c}$ is stable to MI as both wavetrains are stable. (b) $k_{1}=1.5>k_{c}$ (unstable). There is instability for some $K$ at each $k_{2}$ as the $k_{1}$ wavetrain is individually unstable to MI. 
This behaviour is general. For sufficiently long perturbations, the terms of order $\epsilon^{4} K^{4}$ in (6) are negligible and (6) reduces to

$$
\tilde{D}_{1}(\Omega, K) \tilde{D}_{2}(\Omega, K)=0,
$$

where $\tilde{D}_{1}(\Omega, K)=\left(\Omega-K c_{1}\right)^{2}+2 \mu_{1} \epsilon^{2} \Delta_{1} K^{2}|\tilde{A}|^{2}$ and $\tilde{D}_{2}(\Omega, K)$ $=\left(\Omega-K c_{2}\right)^{2}+2 \mu_{2} \epsilon^{2} \Delta_{2} K^{2}|\tilde{B}|^{2}$. The wavetrains effectively decouple, and, since $\mu_{1,2}<0$, (7) has one pair of complex conjugate roots when just one of the $\Delta_{i}$ is negative, i.e., $k_{i}>k_{c}$. If both $\Delta_{1,2}$ are negative, i.e., both $k_{1,2}>k_{c}$, (7) has two pairs of complex conjugate roots and the maximum growth rate is determined by the root with maximum imaginary part. This can be seen in Fig. 2(b) where $k_{1}>k_{c}$ and there is a band of instability for all $k_{2}$ for $K \$ 2$. The coupled wavetrain is unstable if at least one carrier wave is individually unstable. Now consider $k_{2}=k_{c}$ so $\Delta_{2}=0$. Then, Eq. (6) again decouples with the real repeated root $\Omega=-K c_{2}$ plus either a complex conjugate pair and instability for all $K$ if $k_{1}>k_{c}$ as in Fig. 2(b) or two real roots and stable waves for all $K$ if $k_{1}<k_{c}$ as in Fig. 2(a). Since $k_{2}=k_{c}$ gives a double root of (6), the determinant of the quartic changes sign as $k_{2}$ decreases through $k_{c}$ the unstable complex conjugate pair becomes two stable real roots. Similarly, when $k_{1}$ decreases through $k_{c}$, the unstable complex pair becomes two stable real roots. Thus, when both $k_{1,2}<k_{c}$, all roots are real for all parameters and all $K$, and the coupled wavetrain is stable. Similar arguments do not apply to the CNLS for the KdV since $\Delta_{1,2}$ do not vanish for the KdV. The CNLS for the $\mathrm{KdV}$ equation is unstable for all wavenumbers ${ }^{14} k_{1,2}$, and so the consequence of introducing weak rotation-giving the Ostrovsky equation here-is the suppression of this two-wavetrain instability when both wavenumbers are individually stable to MI: a pair of sufficiently long $\left(k_{i}<k_{c}\right)$ co-propagating waves are modulationally stable in the Ostrovsky equation when rotation is present but unstable in the $\mathrm{KdV}$ in the absence of rotation.

\section{Numerical integrations}

To test this CNLS theory, the full Ostrovsky equation was integrated numerically using the method of integrating factors ${ }^{19}$ with a pseudo-spectral Fourier discretization on a periodic domain in $x$ and 4th-order Runge-Kutta Cash$\operatorname{Karp}^{20}$ adaptive time-stepping in $t$. The coefficients of the Ostrovsky equation were set to unity and the initial condition (IC) taken to be

$$
\eta=2 \epsilon\left[\cos \left(k_{1} x\right)+\cos \left(k_{2} x\right)\right],
$$

with small parameter $\epsilon$ set at 0.1 to remain within the range of validity of the CNLS theory. Figs. 3(a) and 3(b) give the initial $(t=0)$ and final $\left(t=t_{f}\right)$ spectra of integrations of the $\mathrm{KdV}$ and Ostrovsky equations for $t_{f}=15000$ and 20000, respectively, with $k_{1,2}<k_{c}$, showing that weak rotation suppresses the instability. Fig. 3(c) gives the initial and final spectra for an integration of the Ostrovsky equation for $t_{f}=7000$ with $k_{1}<k_{c}$ and $k_{2}>k_{c}$ showing that the sidebands on both sides of $k_{1,2}$ have grown through MI. The theory of Sec. II B also predicts the growth rate of the instability seen
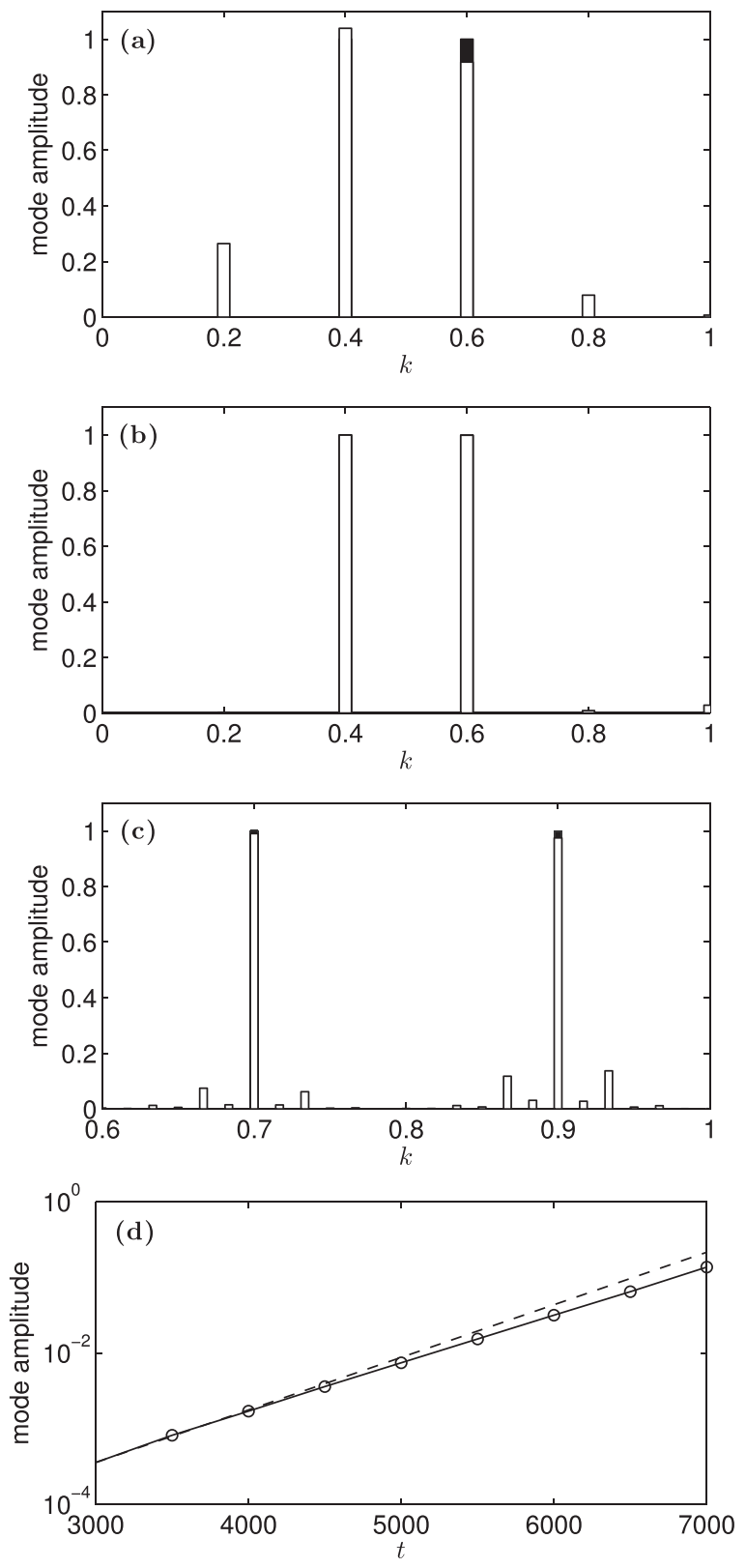

FIG. 3. Integrations of the KdV and Ostrovsky equations with the coefficients set to unity and initial condition (8) with $\epsilon=0.1$. Normalised Fourier spectra showing the spectrum at $t=0$ in black and, superposed in white, the spectrum at the final integration time $t=t_{f}$ : (a) an integration of the $\mathrm{KdV}$ equation with $k_{1}=0.4, k_{2}=0.6$, and $t_{f}=15000$, showing sideband growth; (b) an integration of the Ostrovsky equation for the same wavenumbers $\left(k_{1}=0.4, k_{2}=0.6\right)$ as in (a) and $t_{f}=20000$, showing that rotation has prevented sideband growth; (c) an integration of the Ostrovsky equation for $k_{1}=0.7, k_{2}=0.9$, and $t_{f}=7000$, where one wavenumber lies in the unstable regime and so sidebands grow. (d) A semi-log plot of the time evolution for the $k=0.933$ sideband in (c) (solid line) compared with the theoretical growth rate $\exp \left(\epsilon^{2} 0.16 t\right)$ (dashed line).

in the integrations of the Ostrovsky equation (Fig. 3(c)). As the initial condition in the integration has no initial modulation, the sideband with the largest growth rate should grow first. The infinite wavetrain theory predicts sidebands $k_{i} \pm 0.036$ to have the largest growth rate, whereas in the integration, $k_{i} \pm 0.033$ were found to be most unstable. This is a close agreement, and the difference is a consequence of the periodicity of the computational domain, as the modulation wavelength $2 \pi / \epsilon^{2} K$ must always be a submultiple of the 
domain length. The predicted growth rate for $k_{i} \pm 0.033$ is 0.0016 . Fig. 3(d) compares the height of the spectral peak at $k=0.933$ measured at various times during in the integration with this prediction. The agreement is close with the observed growth rate falling below the predicted linear growth rate only at times such that $\exp \left(\epsilon^{2} \Omega t\right) \geqslant 1$ when the mode amplitude is large and the growth is nonlinearly limited. This close agreement validates the use of (5) in analysing the Ostrovsky equation and differs slightly from the comparison in Onorato et al. ${ }^{14}$ where the analytical growth rate is compared with integrations of the $\mathrm{KdV}$ version of (5) rather than the $\mathrm{KdV}$ itself. It was noted in Sec. II A that the expansion leading to (5) becomes disordered for $\left|k_{1}-k_{2}\right|$ sufficiently small. In practice, this constraint is not strong. Figs. 3(c) and 3(d) show that (5) closely describes the behaviour of the full Ostrovsky equation even when $\left(k_{1}-k_{2}\right)^{2}$ $=0.04$ and $\epsilon=0.1$.

\section{WAVETRAINS WITH THE SAME SPEED}

Since the linear group velocity for the the Ostrovsky equation has a maximum at $k_{c}$, it is possible for wavetrains with different wavenumbers to have the same group velocity, i.e., $k_{1} \neq k_{2}$ but $c_{1}=c_{2}$. This cannot happen in the $\mathrm{KdV}$ equation as its linear group velocity is monotonic in $k$. In discussing the initial value problem for the Ostrovsky equation in the limit of strong rotation, it has been suggested ${ }^{10}$ that an initial KdV soliton breaks up into two co-propagating inertia-gravity wavetrains that can be locally approximated as having constant wavenumbers $k_{+}$with $k_{-}<k_{c}<k_{+}$and the same group velocities $c_{1}=c_{2}=c$ (say). Since $k_{-}<k_{c}$, the $k_{-}$ wavetrain lies in the defocussing regime of the single NLS, and since $k_{+}>k_{c}$, the $k_{+}$wavetrain lies in the focussing regime. ${ }^{16}$ The unstable wavetrain has been proposed as the source of the nonlinear wavepacket that grows in the wavetrain and the carrier wavenumber of the packet estimated by considering the modulational instability of the $k_{+}$wavetrain in isolation. ${ }^{10}$ The analysis of Sec. II allows the accuracy of this approximation to be assessed.

Since both wavetrains have the same group speed $c$, $\epsilon$ can be eliminated directly from (4) by introducing $\zeta=X-c T$ and so moving to the reference frame of the common group speed. The extra transformation leading to (5) is not required in this case and the coupled system is asymptotically consistent with

$$
\begin{aligned}
& \mathrm{i} A_{\tau}+\Delta_{1} A_{\zeta \zeta}+\left(\mu_{1}|A|^{2}+\sigma_{1}|B|^{2}\right) A=0, \\
& \mathrm{i} B_{\tau}+\Delta_{2} B_{\zeta \zeta}+\left(\mu_{2}|B|^{2}+\sigma_{2}|A|^{2}\right) B=0 .
\end{aligned}
$$

The dispersion relation for wavelike disturbances to two constant amplitude wavetrains in (9) is given by (6) with $\epsilon=1$ and $c_{1,2}=0$. The general considerations for roots still hold, and since $k_{+}>k_{c}$, the coupled wavetrain is always unstable. The quartic becomes a simple monic quadratic equation for $\Omega^{2}$. When

$$
\left(2 \mu_{1}|\tilde{A}|^{2}-\Delta_{1} K^{2}\right)\left(2 \mu_{2}|\tilde{B}|^{2}-\Delta_{2} K^{2}\right)>4 \sigma_{1} \sigma_{2}|\tilde{A}|^{2}|\tilde{B}|^{2},
$$

which is always true for sufficiently large $k_{+}$, the roots for $\Omega^{2}$ are real, of opposite signs, and so $\Omega$ is purely imaginary in the unstable mode. The instability grows in situ as for a single wavetrain, giving an absolute instability in the group velocity frame as can be seen in Fig. 4(b) of Ref. 10, where the Gaussian envelope grows while maintaining the same mean in the group velocity frame. When (10) does not hold, the four roots for $\Omega$ are a complex conjugate pair and their negatives. The unstable mode is a propagating growing wave giving a convective instability, raising the possibility, outside the scope of the present analysis that for wavetrains that are not arbitrarily long as those considered here but have finite envelopes like the Gaussian of Fig. 4(b) of Ref. 10, the unstable mode could propagate out of the unstable region before growing to a significant amplitude. Fig. 4(a) shows the maximum growth rate for the $k_{+}$and $k_{-}$wavetrains in the CNLS (dashed line) and for the $k_{+}$wavetrain in isolation in the NLS (solid line). For large $k_{+}$, the CNLS and NLS growth rates converge showing that the uncoupled NLS approximation in Ref. 10 is accurate for sufficiently short carrier waves. Because of the weakening of the interaction term, as shown in Fig. 1, this convergence of coupled to uncoupled growth rates also occurs for arbitrary wavenumbers provided $\left|k_{1}-k_{2}\right|$ is sufficiently large. For $0.8 \lesssim k_{+} \lesssim 1.5$, the coupled and uncoupled growth rates differ suggesting that the presence of the stable $k_{-}$wavetrain significantly affects the growth rate of the $k_{+}$packet. Fig. 4(b) shows that the wavenumber $K_{\max }$ of the modulation with maximum growth rate is also affected by the the second wavetrain. The change in behaviour of the CNLS growth rate at $k_{+} \sim 1.15$ occurs where the fastest growing modulation moves from being an absolute instability as in the uncoupled problem to the convective instability of the coupled problem.
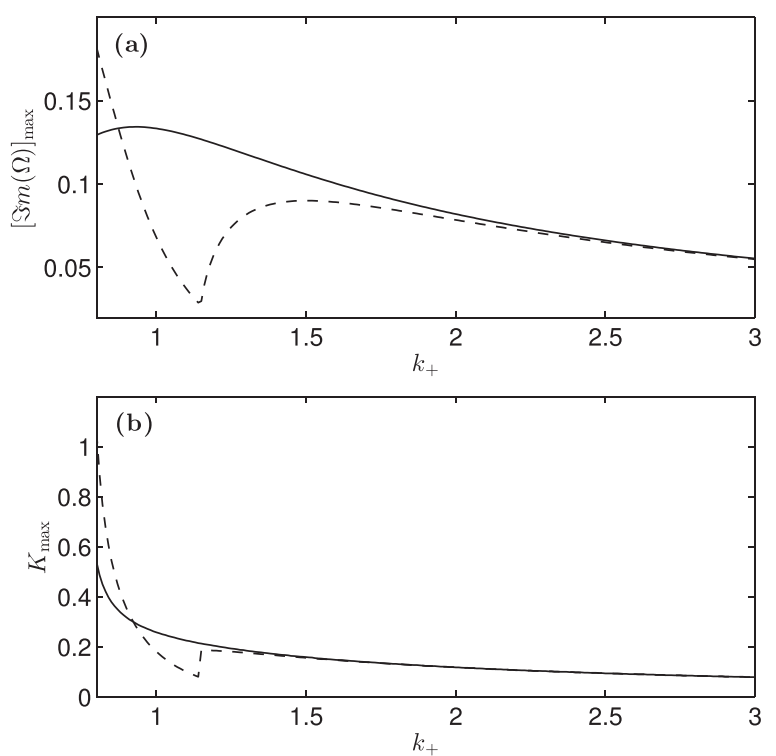

FIG. 4. (a) The maximum growth rate $[\Im m(\Omega)]_{\max }$ for the $k_{+}$wavetrain when considered in isolation in the NLS (solid line) and when considered as co-propagating with $k_{-}$in the CNLS (dashed line). In both cases, $\tilde{A}, \tilde{B}, \alpha, \beta$, $\gamma=1$. (b) The value $K_{\max }$ of the modulation wavenumber $K$ corresponding to the maximum growth rate in (a). The CNLS and NLS theories converge for large $k_{+}$. 


\section{DISCUSSION}

This paper considers the effect of rotation on two copropagating internal wavetrains within the CNLS regime of the Ostrovsky equation. The main effect of rotation compared to the non-rotating $\mathrm{KdV}$ case is the disappearance of the mean flow term due to the zero-mass constraint on solutions of the Ostrovsky equation. As this is the dominant interaction term, MI is suppressed in rotating flows when both wavetrains are individually stable, i.e., for wavenumbers $k_{1,2}<k_{c}$. The CNLS theory is strongly supported by numerical integrations of the full Ostrovsky equation. For $\left|k_{1}-k_{2}\right|$ sufficiently large, the wavetrains can be considered separately in the normal NLS, but when $\left|k_{1}-k_{2}\right|$ is smaller, the interaction must be taken into account and the full CNLS used. Although $\left|k_{1}-k_{2}\right|$ is formally restricted from being too small by the requirement that the amplitude expansion remains well-ordered, integrations of the full underlying Ostrovsky equation show this restriction to be weak in practice. Since the linear group velocity of the Ostrovsky equation achieves its maximum value at finite, non-zero $k_{c}$, two wavetrains can have different wavenumbers, $k_{-}<k_{c}<k_{+}$, but the same linear group velocity. As in the general case, the interaction of the two wavetrains becomes important when $\left|k_{-}-k_{+}\right|$is not large.

This paper has considered the modulational instability of two co-propagating wavetrains; however, a stochastically forced sea could contain more than two distinct wavetrains. As above, background rotation removes the mean flow interaction term, and so it appears that once again the flow is modulationally unstable if and only if at least one of the component wavetrains is modulationally unstable in isolation. The shape of the dispersion relation for the Ostrovsky equation means that there is no frame in which the group velocities of three or more distinct wavetrains coincide and so detailed analysis would follow the more general lines of Sec. II.

${ }^{1}$ L. Ostrovsky and Y. A. Stepanyants, "Do internal solitions exist in the ocean?," Rev. Geophys. 27, 293-310, doi:10.1029/RG027i003p00293 (1989).
${ }^{2}$ R. Grimshaw, Advances in Coastal and Ocean Engineering, edited by P. L.-F. Liu (World Scientific Publishing Company, Incorporated, 1997), Chap. I, pp. 1-30.

${ }^{3}$ K. R. Helfrich and W. K. Melville, "Long nonlinear internal waves," Annu. Rev. Fluid Mech. 38, 395-425 (2006).

${ }^{4}$ J. R. Apel, L. A. Ostrovsky, Y. A. Stepanyants, and J. F. Lynch, "Internal solitons in the ocean and their effect on underwater sound," J. Acoust. Soc. Am. 121, 695-722 (2007).

${ }^{5} \mathrm{~T}$. Stanton and L. Ostrovsky, "Observations of highly nonlinear internal solitons over the continental shelf," Geophys. Res. Lett. 25, 2695-2698, doi:10.1029/98GL01772 (1998).

${ }^{6}$ L. Ostrovsky and Y. Stepanyants, "Nonlinear surface and internal waves in rotating fluids," in Nonlinear Waves 3, Research Reports in Physics, edited by A. Gaponov-Grekhov, M. Rabinovich, and J. Engelbrecht (Springer Berlin Heidelberg, 1990), pp. 106-128.

${ }^{7}$ R. Grimshaw, L. Ostrovsky, V. Shrira, and Y. Stepanyants, "Long nonlinear surface and internal gravity waves in a rotating ocean," Surv. Geophys. 19, 289-338 (1998).

${ }^{8}$ L. A. Ostrovsky, "Nonlinear internal waves in a rotating ocean," Oceanology 18, 199-125 (1978).

${ }^{9}$ R. H. J. Grimshaw, J.-M. He, and L. A. Ostrovsky, "Terminal damping of a solitary wave due to radiation in rotational systems," Stud. Appl. Math. 101, 197-210 (1998).

${ }^{10}$ A. J. Whitfield and E. R. Johnson, "Rotation-induced nonlinear wavepackets in internal waves," Phys. Fluids 26, 056606 (2014).

${ }^{11}$ A. Alias, R. H. J. Grimshaw, and K. R. Khusnutdinova, "Coupled Ostrovsky equations for internal waves in a shear flow," Phys. Fluids 26, 126603 (2014).

${ }^{12}$ A. Alias, R. H. J. Grimshaw, and K. R. Khusnutdinova, "On strongly interacting internal waves in a rotating ocean and coupled Ostrovsky equations," Chaos 23, 023121 (2013).

${ }^{13}$ E. F. Thompson, "Energy spectra in shallow US coastal waters," Tech. Rep. (DTIC Document, 1980).

${ }^{14}$ M. Onorato, D. Ambrosi, A. Osborne, and M. Serio, "Interaction of two quasi-monochromatic waves in shallow water," Phys. Fluids 15, 3871-3874 (2003).

${ }^{15}$ T. B. Benjamin and J. E. Feir, "The disintegration of wave trains on deep water," J. Fluid Mech 27, 417-430 (1967).

${ }^{16}$ R. Grimshaw and K. Helfrich, "Long-time solutions of the Ostrovsky equation," Stud. Appl. Math. 121, 71-88 (2008).

${ }^{17}$ V. E. Zakharov, "Stability of periodic waves of finite amplitude on the surface of a deep fluid," J. Appl. Mech. Tech. Phys. 9, 190-194 (1968).

${ }^{18}$ S. Griffiths, R. Grimshaw, and K. Khusnutdinova, "Modulational instability of two pairs of counter-propagating waves and energy exchange in a two-component system," Physica D 214, 1-24 (2006).

${ }^{19}$ L. N. Trefethen, Spectral Methods in MATLAB (Society for Industrial and Applied Mathematics, 2000), Vol. 10, p. 111.

${ }^{20}$ J. R. Cash and A. H. Karp, "A variable order Runge-Kutta method for initial value problems with rapidly varying right-hand sides," ACM Trans. Math. Software 16, 201-222 (1990). 